\title{
Zur Sinnhaftigkeit einer parallelen Vermittlung von Fachsprachen- und Varietätenkompetenz. Plädoyer für die Institutionalisierung einer studienbegleitenden Zusatzausbildung ,Fachsprache Deutsch mit regionalem Akzent' an tschechischen Hochschulen
}

\section{Boris Blahak}

The article first describes Bavaria's role as the main trading partner of the Czech Republic based on an own Bavarian 'foreign policy', a growing bilateral trading volume and a rising Czech surplus of export. As German is moreover still a widely spread language in companies this economic partnership creates an increased need for (regional specialized) skills in German as a technical language corresponding to a EU-wide trend. These circumstances are faced by a decreasing importance of German in Czech schools: It is true that widely spread but no longer advanced skills can still be expected, particulary as German will be limited to the role as a second foreign language in the future. This situation leads to a big demand for teaching lessons in German as a technical language in adult education. Due to this situation and a present discussion about regionalizing German studies the author pleads for institutionalizing a course of studies - 'German as a technical language with regional focus' - as an additional training focussing on the state of Bavaria as a main region.

Introduction of technical terminology - Bavarian-Czech economic relations - program for additional educational activities - motivation for learning a foreign language - number of people studying German - adult education - business language - regionalization - competence regarding dialects

Der Beitrag arbeitet zunächst die Rolle Bayerns als Haupthandelspartner Tschechiens heraus, die durch eine eigene bayerische,Außenpolitik' und ein wachsendes bilaterales Handelsvolumen bei steigendem tschechischem Exportüberschuss begründet ist. Da Deutsch in Tschechien zudem immer noch eine verbreitete Firmensprache ist, schafft diese Wirtschaftspartnerschaft einen erhöhten Bedarf an (landesspezifischen) Fachsprachen-Kenntnissen in Deutsch, der einem allgemeinen EU-Trend entspricht. Dem steht die sinkende Bedeutung des Deutschen an tschechischen Schulen gegenüber: Hier ist zwar noch von breiten aber nicht mehr fortgeschrittenen Kenntnissen auszugehen, zumal Deutsch zukünftig auf die Rolle einer zweiten Fremdsprache beschränkt ist. Diese Situation führt zu einem großen Deckungsbedarf an Fachsprachenunterricht in der Erwachsenenbildung. Angesichts dieser Situation und der der aktuellen Debatte um eine Regionalisierung der Germanistik erfolgt ein Plädoyer für die Institutionalisierung einer studienbegleitenden Zusatzausbildung ,Fachsprache Deutsch mit regionalem Akzent' mit dem Bundesland Bayern als Zielregion.

Fachsprachenausbildung - $\quad$ Bayerisch-tschechische Wirtschaftsbeziehungen Rahmenbildungsprogramm - Sprachlernmotivation - Deutschlerner-Zahlen Erwachsenenbildung - Firmensprache - Regionalisierung - Varietätenkompetenz. 


\section{Grundlegende Überlegungen bezüglich des Bedarfs einer regional ausgerichteten Fachsprachenausbildung Deutsch innerhalb der tschechischen Auslandsgermanistik}

Ziel der folgenden Studie ist es, die aktuelle Situation des Deutschen als Fremdsprache in Tschechien zu beschreiben und den Befund mit dem Umfang bayerischtschechischer Wirtschaftsbeziehungen zu kontrastieren. Dabei soll gezeigt werden, dass vieles auf einen wachsenden Bedarf an tschechischen Marktteilnehmern mit guten Fachsprachen-Kenntnissen in Deutsch hindeutet, der aufgrund bildungspolitischer Maßnahmen in Tschechien allerdings zunehmend weniger gedeckt werden kann. Angesichts dieser Situation erfolgen Überlegungen zur Sinnhaftigkeit der Institutionalisierung einer studienbegleitenden Zusatzausbildung ,Fachsprache Deutsch mit regionalem Akzent' an tschechischen Hochschulen, die hinsichtlich der besonderen bayerisch-tschechischen Wirtschaftspartnerschaft berufssprachliche Fertigkeiten durch landesspezifische (fachliche wie regionalsprachliche) ZusatzKompetenzen ergänzt.

\section{Der Freistaat Bayern und die Tschechische Republik als Wirtschaftspartner}

2.1. Die besondere, wirtschafts- und regionalpolitisch bestimmte ,Außenpolitik' Bayerns gegenüber dem östlichen Nachbarland Tschechien

Die Einrichtung wirtschaftssprachlicher Regionalstudien mit einem konkreten Bundesland als Zielregion innerhalb der Auslandsgermanistik scheint zunächst vor dem Hintergrund besonderer bilateraler Beziehungen berechtigt, die sich auf der ,Dritten Ebene' der EU, die den Regionen gewisse eigenständige Kompetenzen im Rahmen einer beschränkten, wirtschaftspolitisch geprägten ,Außenpolitik' im Bildungs-, Hochschul- und Wirtschaftsbereich einräumt, zwischen einigen deutscher Bundesländern und eigenständigen Staaten entwickelt haben. Inzwischen ist es hier zu echten Institutionalisierungen gekommen. Dass aus deutscher Sicht direkte Anrainerstaaten dabei bevorzugt werden, erscheint naheliegend. Ein besonderer Nachholbedarf besteht dabei gegenüber den neuen und zukünftigen EU-Staaten in Mittel-, Ost- und Südost-Europa - zum einen aufgrund historischer, aus dem Zweiten Weltkrieg herrührender Wunden und der Jahrzehnte langen hemmenden Auswirkungen des Kommunismus auf nachbarschaftliche Beziehungen, zum anderen aber auch aufgrund neuer, vielversprechender wirtschaftlicher Perspektiven seit 1989.

So verwundert es nicht, dass diese Formen bilateraler Wirtschaftspolitik v. a. in den östlichen und südöstlichen Ländern der Bundesrepublik stark ausgeprägt sind: In Brandenburg etwa genießt die Zusammenarbeit mit Polen Verfassungsrang 
(Art. 2 (1)) und erfährt eine bevorzugte Förderung durch die Landesregierung. Eine eigenständige äußere Wirtschaftspolitik forcieren zudem drei Bundesländer, die offizielle Repräsentanzen (,Büros') bei bilateralen Industrie- und Handelskammern (IHK) im Ausland unterhalten. Hessen, Sachsen und Bayern sind auf diese Weise in der deutsch-ungarischen IHK Budapest gesondert vertreten, Sachsen und Bayern darüber hinaus in der deutsch-tschechischen IHK Prag. Der Freistaat Bayern beweist ein besonderes Engagement, indem er weitere Vertretungen in den IHK in Warschau, Sofia und Zagreb unterhält und selbst in die Ukraine einen Beauftragten im Rahmen der dortigen Delegation der deutschen Wirtschaft entsendet. ${ }^{1}$ Für Bayern scheint Tschechien als Partner dabei schon deswegen eine hervorgehobene Rolle einzunehmen, da es als einziges der genannten Länder direkt an den Freistaat grenzt und mit diesem zudem eng durch mannigfaltige historisch-politische, kulturelle und wirtschaftliche Bezüge verknüpft ist.

Diese staatliche bilaterale Wirtschaftskooperation vollzieht sich auf halb- und nicht-staatlicher Ebene vor dem Hintergrund euroregionaler Zusammenarbeit und wird u. a. durch den Freistaat Bayern, die EU, den Deutschen Akademischen Austauschdienst (DAAD) und den Deutsch-tschechischen Zukunftsfonds sowie durch zahlreiche hochschulpolitische Initiativen und Institutionen gefördert, die sich u. a. um die bilaterale akademische Mobilität, die Vermittlung von Auslandspraktika und den Wissenstransfer bemühen: Im Falle Bayerns ist hier v. a. das akademische Stipendien- und Austauschprogramm des Bayerischen Hochschulzentrums für Mittel, Ost-und Südosteuropa (BayHost) an der Universität Regensburg zu nennen, die mit der Zusatzausbildung Bohemicum (in Kooperation mit der Masaryk-Universität Brno) und den Deutsch-Tschechischen Studien (im Verbund mit der Karls-Universität Prag) ${ }^{2}$ die Annäherung an den Nachbarn Tschechien zudem auch in Form besonderer Ausbildungszweige fördert. Die dem zugrundeliegende Einsicht in die Wichtigkeit guter (wirtschaftlicher) Beziehungen mit der Nachbarregion ist unverkennbar.

\subsection{Zu Umfang und Perspektiven der gegenwärtigen bayerisch- tschechischen Wirtschaftsbeziehungen}

Vor diesem Hintergrund stellt sich die Frage, welche Intensität die gegenwärtige Verflechtung der bayerisch-tschechischen Wirtschaft aufweist, die ein potentielles Betätigungsfeld für tschechische Hochschulabsolventen bietet. Dabei ist aufgrund des Umfangs der Import- und Export-Ströme zwischen Bayern und Tschechien

1 Vgl. die Homepages der jeweiligen IHK (H: http://www.duihk.hu - CZ: http://tschechien.ahk. de - PL: http://www.ihk.pl - BG: http://bulgarien.ahk.de - HR: http://kroatien.ahk.de - UA: http://www.dihk.com.ua).

2 S. die jeweiligen Homepages unter: www.bayhost.de; www.bohemicum.de; http://www.bohemicum. de/studium/deutsch-tschechische-studien.html. 
zu konstatieren, dass Bayern ${ }^{1}$ unbestritten einer der Haupthandelspartner seines östlichen Nachbarn ist und das bilaterale Handelsvolumen nicht erst seit Tschechiens EU-Beitritt von 2004 rasant angewachsen ist.

Als aufschlussreich erweist sich auch ein statistischer Vergleich Bayerns mit dem potentiell größten und direkten deutschsprachigen Konkurrenten im Wettbewerb um den tschechischen Markt: der Republik Österreich. Diese scheint mit dem Freistaat bezüglich westlich-marktwirtschaftlicher Tradition, territorialer Ausdehnung, Bevölkerungszahl und volkswirtschaftlicher Leistung (BIP, Inflation, Arbeitslosigkeit) ${ }^{2}$ sowie der Nachbarschaft zu Tschechien durchaus vergleichbar disponiert und verfolgt zudem wie die Bundesrepublik und mit ihr (und damit mittelbar auch mit Bayern) konkurrierend nach außen eine vergleichbare Sprachförderungs- und Kulturpolitik ${ }^{3}$, die besonders Akademiker und gut ausgebildete ausländische Schlüsselkräfte anziehen soll.

Obwohl man aufgrund alter historisch-kultureller Beziehungen (k. u. k. Monarchie) eine deutlich stärkere wirtschaftliche Orientierung tschechischer Firmen auf Österreich hin erwarten könnte, fällt der direkte Vergleich des bilateralen Handelsvolumens mit Tschechien sichtbar zu Gunsten Bayerns aus, wie die folgende Statistik zum Import/Export im Zeitraum 2004-2007 verdeutlicht:

\begin{tabular}{|c|c|c|c|c|c|c|c|c|c|c|}
\hline \multicolumn{11}{|c|}{ Export nach Tschechien ${ }^{4}$} \\
\hline \multirow[t]{2}{*}{ Jahr } & \multicolumn{2}{|c|}{$\begin{array}{l}\text { Export-Volumen } \\
\text { nach CZ }\end{array}$} & \multicolumn{2}{|c|}{$\begin{array}{c}\text { Platzierung von } \\
\text { CZ im EU-25- } \\
\text { Vergleich }\end{array}$} & \multicolumn{2}{|c|}{$\begin{array}{l}\text { Platzierung von CZ } \\
\text { im EU-105-Vergleich }\end{array}$} & \multicolumn{2}{|c|}{$\begin{array}{l}\text { Zuwachs } \\
\text { gegen-über dem } \\
\text { Vorjahr (EU-25) }\end{array}$} & \multicolumn{2}{|c|}{$\begin{array}{c}\text { Anteil am ges. } \\
\text { EU-25-Export- } \\
\text { Volu-men des } \\
\text { Landes }\end{array}$} \\
\hline & Bayern & Österr. & Bayern & Österr. & Bayern & Österr. & Bayern & Österr. & Bayern & Österr. \\
\hline 2004 & $\begin{array}{l}3.320 \\
\text { Mil. } €\end{array}$ & $\begin{array}{l}2.752 \\
\text { Mil. } €\end{array}$ & 9. & 6. & $\begin{array}{l}\text { 2. (nach } \\
\text { Ungarn) }\end{array}$ & $\begin{array}{l}\text { 2. (nach } \\
\text { Ungarn) }\end{array}$ & - & - & $\begin{array}{c}4,71 \\
\%\end{array}$ & $\begin{array}{c}4,16 \\
\%\end{array}$ \\
\hline 2005 & $\begin{array}{l}3.538 \\
\text { Mil. € }\end{array}$ & $\begin{array}{l}2.925 \\
\text { Mil. } €\end{array}$ & 9. & 6. & $\begin{array}{l}\text { 2. (nach } \\
\text { Ungarn) }\end{array}$ & $\begin{array}{l}\text { 2. (nach } \\
\text { Ungarn) }\end{array}$ & $\begin{array}{c}+9,5 \\
\% \\
\end{array}$ & $\begin{array}{c}+6,3 \\
\%\end{array}$ & $\begin{array}{c}4,60 \\
\%\end{array}$ & $\begin{array}{c}, 22 \\
\%\end{array}$ \\
\hline 2006 & $\begin{array}{l}4.215 \\
\text { Mil. } €\end{array}$ & $\begin{array}{l}3.384 \\
\text { Mil. } €\end{array}$ & 9. & 6. & $\begin{array}{l}\text { 2. (nach } \\
\text { Ungarn) }\end{array}$ & $\begin{array}{l}\text { 2. (nach } \\
\text { Ungarn) }\end{array}$ & $\begin{array}{c}+19,1 \\
\%\end{array}$ & $\begin{array}{c}+15,7 \\
\%\end{array}$ & $\begin{array}{c}4,87 \\
\%\end{array}$ & $\begin{array}{c}4,47 \\
\%\end{array}$ \\
\hline 2007 & $\begin{array}{l}4.840 \\
\text { Mil. } €\end{array}$ & $\begin{array}{l}4.088 \\
\text { Mil. } €\end{array}$ & 8. & 4. & 1. & 1. & $\begin{array}{c}+14,8 \\
\%\end{array}$ & $\begin{array}{c}+20,8 \\
\%\end{array}$ & $\begin{array}{c}5,05 \\
\%\end{array}$ & $\begin{array}{c}4,92 \\
\%\end{array}$ \\
\hline
\end{tabular}

1 Der Freistaat ist seit 2004 im Schnitt zu 19-20\% an der Einfuhr und zu 16-17 \% an der Ausfuhr des bundesdeutschen Außenhandels beteiligt (vgl. AB: 2005, 4; AB: 2006, 4; AB: 2007, 4).

2 Als Exporteure befanden sich beide Länder 2007 weltweit unter den Top 25, als Importeure auf Platz 19 (Bayern) bzw. 21 (Österreich) (vgl. WH: 2007, 1; AB: 2007, 4).

3 Im Detail Goethe-Institute ,vs. ' Österreich-Institute und Österreichische Kultur-Foren; DAAD und BayHost ,vs. ' Österreichischer Akademischer Austauschdienst (ÖAD) und Österreich-Kooperation (ÖK).

4 Die Zahlen zum Export nach Tschechien entstammen AB $(2005,5 ; 2006,5 ; 2007,5)$ und WKO (2007a, 1).

5 Mit ,EU-10‘ werden im Folgenden die zehn EU-Beitrittsländer von 2004 bezeichnet. 
So fiel das Volumen des Exports bayerischer Unternehmen nach Tschechien im untersuchten Zeitraum im Schnitt um 20,6-24,6 \% höher aus als bei ihren österreichischen Konkurrenten. Bei einem etwa vergleichbaren jährlichen Zuwachs der Exporte nach Tschechien und einer identischen Spitzenstellung Tschechiens in der Export-Statistik beider Länder im direkten Vergleich mit den anderen EU-10Ländern war in Bayern der Anteil der Ausfuhren nach Tschechien am gesamten eigenen EU-25-Ausfuhrvolumen auch sichtbar (um 0,13-0,55 Prozentpunkte) höher als die Vergleichswerte in Österreich (s. Tabelle o.).

Noch deutlicher zeigt die Import-Statistik die größere Bedeutung des Handelspartners Tschechien für Bayern (und umgekehrt) im Vergleich zu Österreich:

\begin{tabular}{|c|c|c|c|c|c|c|c|c|c|c|}
\hline \multicolumn{11}{|c|}{ Import aus Tschechien ${ }^{1}$} \\
\hline \multirow[t]{2}{*}{ Jahr } & \multicolumn{2}{|c|}{$\begin{array}{c}\text { Import-Volumen } \\
\text { aus CZ }\end{array}$} & \multicolumn{2}{|c|}{$\begin{array}{l}\text { Platzierung von } \\
\text { CZ im EU-25- } \\
\text { Vergleich }\end{array}$} & \multicolumn{2}{|c|}{$\begin{array}{l}\text { Platzierung von } \\
\text { CZ im EU-10- } \\
\text { Vergleich }\end{array}$} & \multicolumn{2}{|c|}{$\begin{array}{l}\text { Zuwachs } \\
\text { gegen-über dem } \\
\text { Vorjahr (EU-25) }\end{array}$} & \multicolumn{2}{|c|}{$\begin{array}{c}\text { Anteil am ges. } \\
\text { EU-25-Import- } \\
\text { Volu-men des } \\
\text { Landes }\end{array}$} \\
\hline & Bayern & Österr. & Bayern & Österr. & Bayern & Österr. & Bayern & Österr. & Bayern & Österr. \\
\hline 2004 & $\begin{array}{l}4.568 \\
\text { Mil. } €\end{array}$ & $\begin{array}{l}2.886 \\
\text { Mil. } €\end{array}$ & 5. & 4. & 1. & 1. & - & - & $\begin{array}{c}8,26 \\
\%\end{array}$ & $\begin{array}{c}4,10 \\
\%\end{array}$ \\
\hline 2005 & $\begin{array}{l}4.697 \\
\text { Mil. € }\end{array}$ & $\begin{array}{l}3.189 \\
\text { Mil. € }\end{array}$ & 6. & 4. & 1. & 1. & $\begin{array}{c}+2,8 \\
\% \\
\end{array}$ & $\begin{array}{c}+10,5 \\
\%\end{array}$ & $\begin{array}{c}8,14 \\
\%\end{array}$ & $\begin{array}{c}, 35 \\
\%\end{array}$ \\
\hline 2006 & $\begin{array}{l}6.201 \\
\text { Mil. } €\end{array}$ & $\begin{array}{l}3.354 \\
\text { Mil. } €\end{array}$ & 4. & 4. & 1. & 1. & $\begin{array}{c}+32,0 \\
\%\end{array}$ & $\begin{array}{c}+5,2 \\
\%\end{array}$ & $\begin{array}{c}9,22 \\
\%\end{array}$ & $\begin{array}{c}4,31 \\
\%\end{array}$ \\
\hline 2007 & $\begin{array}{l}7.661 \\
\text { Mil. € }\end{array}$ & $\begin{array}{l}3.638 \\
\text { Mil. € }\end{array}$ & 3. & 4. & 1. & 1. & $\begin{array}{c}+23,5 \\
\%\end{array}$ & $\begin{array}{c}+8,5 \\
\%\end{array}$ & $\begin{array}{c}10,33 \\
\%\end{array}$ & $\begin{array}{c}4,27 \\
\%\end{array}$ \\
\hline
\end{tabular}

Demnach importierten bayerische Firmen 2004-2007 jährlich Waren in einem um 58,3-110\% höheren Wert aus Tschechien als dies österreichische Unternehmen taten. Bei einer identischen Spitzenstellung im EU-10-Vergleich hat Tschechien im Ranking der EU-25-Importpartner in Bayern als Nr. 3 inzwischen einen besseren Stand als in Österreich (Nr. 4). Der Anteil der jährlichen Einfuhren aus Tschechien am gesamten EU-Import war zudem in Bayern im Schnitt etwa doppelt so groß (um 3,79-6,06 Prozentpunkte höher) als in Österreich (s. Tabelle o.). Damit ist Tschechien einer der wenigen Wirtschaftspartner Bayerns, der einen seit 1991 fast stetig wachsenden Ausfuhr-Überschuss gegenüber dem Freistaat vorweisen kann (vgl. DWB: 2008, 10).

Diese lebhaften bilateralen Wirtschaftsbeziehungen spiegeln sich auch im Verzeichnis der Mitglieder der Deutsch-tschechischen Industrie- und Handelskammer (vgl. MDTHK: 2007): Von den 64 Unternehmen, die einen Hauptsitz in Deutschland angaben, sind $20^{2}(31,3 \%)$ in Bayern ansässig, wodurch

1 Die Zahlen zum Import aus Tschechien sind AB (2005, 6; 2006, 6; 2007, 6) und WKO (2007b, 1) entnommen.

2 Darunter u. a. das Bayerische Wirtschaftsministerium, Bayern Handwerk International und die NürnbergMesse GmbH. 
ein überproportionales Handelsinteresse bayerischer Firmen an Tschechien dokumentiert wird. Auch zu den 438 Mitgliedern mit Hauptsitz in Tschechien gehören z. T. Niederlassungen bayerischer bzw. bayerisch-tschechischer Betriebe. Die BMW-Vertriebs GmbH und Frankenland s. r. o. mögen stellvertretend für diese stehen. 2006 konstatierte der damalige bayerische Wirtschaftsminister Huber:

„[I]n Tschechien investieren und produzieren mittlerweile mehr als 300 bayerische Firmen und drei Viertel der hierzulande tätigen deutschen Großunternehmen komm[en] ebenfalls aus Bayern. Aber auch wenn Tschechien für den Freistaat der größte Wirtschafts- und Handelspartner unter den neuen EU-Ländern aus Mittel- und Osteuropa ist, so seien noch längst nicht alle Reserven ausgeschöpft" (zit. nach MARTIN: 2006).

Gleichzeitig gab es nach Angaben von Markus Wittmann, Exekutiv-Direktor von Invest in Bavaria, bereits ca. 70 tschechische Firmen, die in Deutschland investiert hatten. In Bayern waren damals z. B. im Raum Regensburg SoftwareUnternehmen mit Kooperationen und Firmen aus dem Automobilbereich tätig. Die voraussichtliche Aufhebung der eingeschränkten Freizügigkeit für Arbeitnehmer aus den EU-10-Ländern im Jahre 2011 wird den Zugang zum deutschen (und hier v. a. zum bayerischen) Arbeitsmarkt für tschechische Arbeitnehmer weiter vereinfachen.

\subsection{Zwischenfazit I: Die besondere bayerisch-tschechische Wirtschaftspartnerschaft schafft in Tschechien einen erhöhten Bedarf an (landesspezifischen) Fachsprachen-Kenntnissen in Deutsch}

Als Ergebnis von Kap. 2 lässt sich resümieren, dass sich aufgrund des gestiegenen wirtschaftlichen Engagements Bayerns gegenüber Tschechien und der sich zunehmend intensivierenden bilateralen Wirtschaftsverflechtung v. a. vor dem Hintergrund des Export-Überschusses der tschechischen Seite und der 2011 in Kraft tretenden uneingeschränkten Freizügigkeit für tschechische Arbeitnehmer ein erhöhter Bedarf an tschechischen Marktteilnehmern mit guten fachsprachlichen Deutsch-Kenntnissen und landesspezifischen Zusatzkompetenzen ergibt. Nicht zuletzt hat schon VANDERMEEREN $(1998,67)$ darauf hingewiesen, die meisten Verfasser von Bedarfsanalysen verträten die Meinung, dass Unternehmen sich sprachlich an ihre Absatzmärkte anpassen und „daß die Beherrschung der Sprache des Geschäftspartners (vor allem des Kunden) ein Wettbewerbsvorteil ist." Dies wäre im Falle Tschechiens bezüglich des Absatzpartners Bayern das Deutsche. Dieser Bedarf erhöht sich noch durch den Umstand, dass man trotz lokaler Bemühungen (s. Kap. 2.1) in Bayern noch lange nicht von verbreiteten Tschechisch-Kenntnissen auf Verhandlungsniveau ausgehen kann und das Englische, wie noch gezeigt werden wird, (noch) nicht die vielfach prognostizierte Rolle im deutsch-tschechischen Wirtschaftsverkehr eingenommen hat. 


\section{Zur gegenwärtigen Situation des Fremdsprachenunterrichts Deutsch in Tschechien}

Die Feststellung, dass die engen bayerisch-tschechischen Wirtschaftsbeziehungen einen großen Bedarf an tschechischen Fachkräften mit Deutsch-Kenntnissen erwarten lassen, wirft die Frage auf, wie groß das Interesse an Deutsch als Fremdsprache in Tschechien tatsächlich ist und ob eine entsprechende Nachfrage nach (berufsbezogenem) Deutschunterricht gedeckt werden kann.

\subsection{Umfang und Art einer generellen Sprachlernmotivation in Tschechien}

Eine repräsentative Erhebung des Eurobarometer (Nr. 243 / Wave 64.3) versuchte im Jahre 2005 die Einstellung der EU-Bürger zu Fremdsprachen zu ermitteln. Bezüglich der allgemeinen Motivation, fremde Sprachen zu lernen, ließen sich in Tschechien folgende Trends ermitteln: $33 \%$ der Befragten gaben an, einen FremdsprachenErwerb oder die Verbesserung vorhandener Fremdsprachen-Kenntnisse innerhalb des nächsten Jahres anzustreben. Damit gehörte Tschechien nach Lettland und der Slowakei zu den EU-Ländern mit den stärksten Lern-Ambitionen (vgl. EK: 2006, 25, 119). Gleichzeitig lag Tschechien in einem für die gesamte EU festgestellten Trend, die persönliche Motivation zum Erwerb einer Fremdsprache mit praktischen Nützlichkeitsaspekten zu verknüpfen (vgl. DAF: 2006, 5), der eine wachsende Bereitschaft indiziert, in anderen europäischen Ländern zu arbeiten oder zu studieren, und besonders signifikant für die EU-Beitrittsländer von 2004 zutrifft (vgl. EK: 2006, 36-37, 45-46). Unter den persönlichen Hauptgründen, eine neue Fremdsprache zu lernen, wurden dabei in Tschechien folgende karrierebezogene Gründe überdurchschnittlich oft oder zumindest im Bereich des EU-25Durchschnitts genannt (vgl. EK: 2006, 36, 64, 105):

- Um sie bei der Arbeit zu verwenden (incl. Geschäftsreisen im Ausland): $34 \%$ (EU-25: $32 \%$; EU-10: $31 \%$ )

- Um in der Lage zu sein, in einem anderen Land zu arbeiten: $34 \%$ (EU-25: 27 \%; EU-10: $35 \%$ )

- Um (im eigenen Land) eine bessere Arbeit zu erhalten: $23 \%$ (EU-25: 23 \%; EU-10: 28 \%)

- Um in der Lage zu sein, in einem anderen Land zu studieren: $16 \%$ (EU-25: $14 \%$; EU-10: $14 \%$ )

Innerhalb der großen Mehrheit der Europäer (73\%), die glauben, junge Menschen sollten Fremdsprachen lernen, um ihre Berufsmöglichkeiten zu vergrößern, liegen die Tschechen mit $72 \%$ etwa im Bereich des Durchschnitts (vgl. EK: 2006, 102). Bei der Frage „Welcher der folgenden Umstände würde Sie mit signifikant 
höherer Wahrscheinlichkeit zum Lernen einer Fremdsprache oder zur Verbesserung Ihrer Kompetenzen in ihr veranlassen?" wurde die Antwort „Wenn dies zu einer Beförderung bzw. zu besseren Karriereperspektiven führen würde" mit $28 \%$ in Tschechien sogar EU-weit am häufigsten gegeben (vgl. EK: 2006, 124).

Konstatieren lässt sich demnach für die erwachsene tschechische Bevölkerung eine hohe Motivation, Fremdsprachen zu lernen, die in zunehmendem Maße mit beruflichen Aspekten verknüpft wird und somit tendenziell auf einen berufsbezogenen Fremdsprachenunterricht (,Wirtschaftssprache') abzielt. CHRIST $(2002,26)$ nennt entsprechend in seinen Gedanken zu den neuen Dimensionen des Fremdsprachenunterrichts im 21. Jahrhundert u. a. die Bedeutsamkeit der Kategorie des ,lebensbegleitenden Lernens', zu welcher der berufsvorbereitende/begleitende und fachsprachliche Fremdsprachenunterricht gehört. Entsprechende Unterrichtsangebote erfahren heute in fremdsprachlichen Veranstaltungen jeglicher Art einen erkennbaren Zuwachs.

\subsection{Die gegenwärtige Einstellung der Tschechen zur Deutschen Sprache}

Bezüglich des Deutschen ergab das Eurobarometer in Tschechien folgendes Bild: $55 \%$ der Befragten nannten Deutsch als eine von zwei Fremdsprachen, die als die nützlichsten für die eigene persönliche Entwicklung und Karriere eingestuft wurden (Platz 2 nach Englisch mit 70 \%). Damit liegt Tschechien gemeinsam mit Ungarn und Dänemark europaweit auf Platz 4 und deutlich über dem E-25- (22 \%) bzw. E-10-Durchschnitt (48 \%) (vgl. EK: 2006, 30-32, 95). Auf die Frage, welche zwei Fremdsprachen Kinder in der EU lernen sollten, nannten $66 \%$ der Tschechen das Deutsche (Platz 2 nach Englisch mit 89 \%). Mit diesem Anteil lagen sie EU-weit auf Platz 6, wiederum weit über dem EU-25-Schnitt von 28 \% (vgl. EK: 2006, 98).

Dass man auch heute noch mit einer relativ weiten Verbreitung von DeutschKenntnissen in der tschechischen Gesellschaft rechnen kann, belegt auch eine Umfrage des Tschechischen Zentrums für Meinungsforschung von 2003, der zufolge $40 \%$ der Befragten angaben, über Deutschkenntnisse zu verfügen, wogegen sich nur $27 \%$ Englischkenntnisse attestierten. Auch wenn man, wie NEKULA/ ŠICHOVÁ (2004) einschränkend hinzufügen, die Qualität der selbstattestierten Sprachkenntnisse wohl zurückhaltend beurteilen müsse, sind diese Zahlen doch beachtlich.

2005 glaubten 28 \% der vom Eurobarometer befragten Tschechen, Deutsch gut genug zu sprechen, um ein Gespräch führen zu können, was die Tschechische Republik neben der Slowakei und Ungarn zu einem von nur drei Ländern in der EU macht, in welchen es immer noch wahrscheinlicher ist, im Alltag auf Deutschals auf Englisch-Sprecher (24\%) zu treffen (vgl. EK: 2006, 152). Von diesen Deutschsprechern stuften $19 \%$ ihr Deutsch als sehr gut, $52 \%$ als gut ein, $30 \%$ gaben an, über Grundkenntnisse zu verfügen (vgl. EK: 2006, 155). 
Festzuhalten ist für Tschechien also an sich eine im EU-Vergleich überdurchschnittlich positive Einstellung zur deutschen Sprache. Dass diese vermutlich mit der europaweit wachsenden Tendenz zusammenhängt, die persönliche Motivation zum Fremdsprachen-Erwerb mit Nützlichkeitsaspekten zu verknüpfen (vgl. Kap. 3.1), tut dem keinen Abbruch.

\subsection{Die Folgen des EU-Trends hin zu Englisch und der neuen tschechischen Rahmengesetzgebung für Fremdsprachen für das Fach Deutsch an Schulen in Tschechien}

Über die Entwicklung der Deutschlernerzahlen und die institutionellen Rahmenbedingungen des Deutschunterrichts in Tschechien geben die StADaFErhebungen der Jahre 2000 und 2005 Auskunft. Hier manifestiert sich ein sichtbarer Rückgang der Zahlen der Lerner und Unterrichtseinrichtungen, die aber im EUVergleich auf einem immer noch hohen Niveau liegen. Neben dem allgemeinen Englisch-Trend hat wohl auch das Rahmenbildungsprogramm des Tschechischen Schulministeriums (RVP: 2008) diesen Rückgang mit verursacht - trotz der relativ positiven Einstellung im Lande gegenüber dem Deutschen (vgl. Kap. 3.2).

Dieses neue, 2004 verabschiedete und seit dem Schuljahr 2007/08 gültige Schulgesetz wirft seine Schatten voraus: Es schreibt Englisch im Prinzip als erste Fremdsprache an allen Schularten fest. Deutsch und andere Fremdsprachen zählen seither nur noch zu den ,ergänzenden Bildungsbereichen', die von der 7. bis zur 9. Klasse als Pflichtwahlfächer im Umfang von nur zwei Wochenstunden unterrichtet werden. Als Abschlussniveau wird lediglich Niveau A1 des Gemeinsamen Europäischen Referenzrahmens anvisiert (vgl. KOCUMOVÁ: 2008). Durch das neue Schulgesetz ist

„[...] unklar geworden, wie eine Schule zwei Fremdsprachen gleich gut vermitteln soll. Außerdem meinen Eltern sehr oft, sie müssten sich für eine und damit gegen eine andere Fremdsprache entscheiden. Unserer Meinung nach sollte man sich sowohl für Englisch als auch für eine weitere Fremdsprache entscheiden und in Tschechien wäre das dann sinnvollerweise Deutsch“ (Hans Simon-Pelanda zit. nach SILLNER 2006).

So ist Deutsch an tschechischen Schulen seither in der Regel zweite Fremdsprache. Nur an Schulen mit erweitertem Fremdsprachenunterricht kann Deutsch ab der 1. oder 3. Klasse gleichzeitig mit dem Englischen oder als 2. Fremdsprache ab der 5. bzw. 6. Klasse gelernt werden. ${ }^{1}$

1 Hierzu gehören die 22 Schulen, welche das Deutsche Sprachdiplom II der Kultusminister-Konferenz anbieten, das die sprachliche Zugangsvoraussetzung zu einem Hochschulstudium in Deutschland darstellt (vgl. DSD: 2007). 


\subsection{Deutsch als Fremdsprache in Tschechien in Zahlen}

Dieses neue Rahmenbildungsprogramm wird den im Folgenden beschriebenen rückläufigen Trend bezüglich der Verbreitung von Deutsch-Kenntnissen in Tschechien noch verstärken: Im Jahre 2000 rangierte Tschechien in Bezug auf die Deutschlerner in absoluten Zahlen mit 799.071 Lernern noch auf Platz 6 weltweit (2. in der EU-25). 2005 fiel es mit 565.255 Lernern auf Platz 7 zurück (3. in der EU25) - ein Minus von 29,3\%. Hinsichtlich der Zahl der Deutschlerner im Verhältnis zur Einwohnerzahl war Tschechien 2000 mit 7,8 \% sogar weltweit der Spitzenreiter; 2005 büßte das Land mit 5,5 \% drei Plätze ein (vgl. DAF 2003: 2; 2006: 2).

Noch relativ moderat machte sich der Schwund an Deutschlernern an tschechischen Schulen bemerkbar (vgl. DAF: 2003, 16; DAF: 2006, 14): Die Anzahl der Deutsch anbietenden Schulen sank von 7500 (2000) auf 4367 (2005). Die Zahl ihrer Deutschlerner ging entsprechend um 20,4 \% von 624.071 (2000) auf 496.865 (2005) zurück. Wesentlich dramatischer stellt sich die Situation an den Hochschulen dar (vgl. DAF: 2003, 24; DAF: 2006, 14): Hier war 1995 bis 2000 noch ein Anstieg der Studierenden-Zahlen im Bereich der Germanistik und der Deutschlehrerausbildung wie auch in studienbegleitenden DaF-Kursen zu verzeichnen gewesen. Von 2000 bis 2005 ging die Zahl der Hochschuleinrichtungen mit DaF-Unterricht von 189 um 67,7 \% auf 61 zurück. Die Zahl der Germanistik und Lehramt Deutsch Studierenden fiel von 5000 um 80,9\% auf 954. Etwas weniger stark - wenn auch deutlich - sank dagegen gleichzeitig die Zahl der studienbegleitend Deutsch Lernenden von 120.000 um $71,3 \%$ auf 34.436. Am relativ stabilsten erwies sich demgegenüber der Bereich der Erwachsenenbildung (vgl. DAF: 2003, 32; DAF: 2006, 14): Hier reduzierte sich die Zahl der DaF lehrenden Institutionen von 550 auf 330, die Zahl der an diesen Institutionen Deutsch Lernenden fiel um ,lediglich‘ $37 \%$ von 52.395 (2000) auf 33.000 (2005).

Hier lässt sich resümieren, dass Deutsch trotz rückläufiger Lerner-Zahlen in Tschechien immer noch - begründet durch die unmittelbare Nachbarschaft sowie enge wirtschaftliche und kulturelle Kontakte zum deutschen Sprachraum - mit Abstand die wichtigste Fremdsprache nach Englisch ist und das Land im EUVergleich immer noch eine große Zahl an Deutschlernern im Sekundärschulbereich aufweist, deren Sprachniveau aufgrund des vom Umfang her stark reduzierten Unterrichts der zweiten Fremdsprache, die Deutsch inzwischen fast ausschließlich ist, jedoch im Schnitt als nicht fortgeschritten betrachtet werden muss.

Dass viele aus berufsbezogenen Gründen dieses Defizit später im Rahmen ihrer (hochschulischen) Berufsausbildung auszugleichen versuchen, belegen die stabileren Zahlen der Deutschlerner in der Erwachsenenbildung. Während im Rahmen des EU-weiten Trends hin zur Fremdsprache Englisch die Bedeutung der ,klassischen “ Auslandsgermanistik in Zukunft deutlich schwinden wird, erweist sich ein Bedarf an berufsbegleitender fachsprachlicher Deutschausbildung als mittelfristig gegeben. 
Diese Erscheinung scheint den oben skizzierten tschechischen und EU-weiten Trend hin zum Nutzwert des Fremdsprachenerwerbs (vgl. Kap. 3.1) zu bestätigen. Man kann demnach festhalten, dass sich in Tschechien im tertiären Bildungsbereich eine Gewichtsverlagerung weg vom germanistischen Fachstudium hin zu einem stärker berufsorientierten Deutschunterricht abzeichnet.

\subsection{Deutsch als Firmensprache in deutsch-, österreichisch- und schweizerisch-tschechischen Unternehmen}

Der Trend hin zur Fachsprache Deutsch - vorzüglich im Bereich der Erwachsenenbildung - scheint auch durch den Umstand mit bedingt, dass das Deutsche als Firmensprache in national gemischten Unternehmen immer noch eine überraschend starke Position einnimmt: Schon die repräsentative Umfrage von VANDERMEEREN $(1998,153)$ ergab, dass sich deutsche Firmen bei Geschäftskontakten mit tschechischen Partnern in 71 \% der Fälle nur bzw. regelmäßig des Deutschen bedienten, lediglich in 50,5 \% der Fälle nur bzw. regelmäßig des Englischen. ${ }^{1}$

Neuere Aussagen über das Deutsche als offizielle Firmensprache machen die Zahlen, die NEKVAPIL/NEKULA/ŠICHOVÁ (2005, 18) in Bezug auf die Sprachenwahl in deutsch-, österreichisch- und schweizerisch-tschechischen Betrieben ermittelt haben: ${ }^{2}$ Entgegen der verbreiteten Ansicht, dass in solchen Firmen vorwiegend auf Englisch kommuniziert werde, gaben $55 \%$ der befragten Betriebe, sofern sie eine offizielle Betriebssprache festgelegt hatten, an, dass diese Deutsch sei. Weitere $20 \%$ nannten Deutsch als eine von zwei Firmensprachen. In $75 \%$ der Firmen hatte Deutsch also den Status einer Betriebssprache. ${ }^{3}$ Man kann daher davon ausgehen, dass Deutsch-Kenntnisse in tschechischen Firmen, die Kontakte mit Unternehmen aus dem deutschsprachigen Raum unterhalten, nach wie vor eine gefragte Ressource sind und einen nicht zu unterschätzenden Wettbewerbsvorteil darstellen.

\subsection{Zwischenfazit II: Breite aber meist nicht fortgeschrittene (fachsprachliche) Deutschkenntnisse schaffen einen Bedarf im Bereich der Erwachsenenbildung}

Aus Kap. 3 ergibt sich: Dem wachsenden Bedarf an Hochschulabsolventen mit guten Wirtschaftsdeutsch-Kenntnissen und landesspezifischem Zusatzwissen, der

1 Das jeweilige ,regelmäßig' ergibt hier Überschneidungen.

$217 \%$ von knapp 2000 befragten Firmen antworteten; $52 \%$ davon hatten eine offizielle Betriebssprache.

3 Die genauen Zahlen lauten: Deutsch offizielle Betriebssprache (55\%) - Englisch offizielle Betriebssprache (16\%) - Deutsch und Englisch offizielle Betriebssprache (15\%) - Tschechisch offizielle Betriebssprache (9\%) - Tschechisch und Deutsch offizielle Betriebssprache (5\%). 
sich aus den intensiven bayerisch-tschechischen Wirtschaftsbeziehungen und der stabilen Position des Deutschen als Firmensprache in Tschechien ergibt, stehen ein qualitativ sinkendes Angebot an Deutschunterricht an allgemeinbildenden Schulen und rückläufige Lernerzahlen in klassisch-philologischen HochschulStudiengängen gegenüber. Hier macht sich der EU-weite Trend zum Englischen bemerkbar, wie er sich auch im neuen Rahmenbildungsprogramm des tschechischen Schulministeriums niederschlägt. Grundkenntnisse in Deutsch werden dagegen auch künftig (allerdings auf deutlich niedrigerem Niveau als bisher) in breiten Bevölkerungsschichten vorhanden sein. Beides zusammen (ein großer praktischer Bedarf an Deutsch-Kenntnissen bei einer schwindenden Bedeutung des schulischen Deutschunterrichts) generiert einen starken Nachholbedarf im Rahmen der Erwachsenenbildung.

\section{Die gegenwärtige wissenschaftliche Debatte über eine Regionalisierung innerhalb der (Auslands-) Germanistik}

Zeitgleich zu dem gestiegenen Interesse am Deutschen als fachbezogene Fremdsprache (vgl. Kap. 3.4 - 3.5) wird eine Regionalisierung der Germanistik in Forschung und Lehre diskutiert: Ausgelöst durch die Anfang der 1990er Jahre von wissenschaftlicher Seite wahrgenommene ,Dialektrenaissance in Deutschland (vgl. MATTHEIER: 1994, 413) brachte das Forschungsfeld DaF im Rahmen sprachpolitischer Debatten immer häufiger Studien hervor, die nicht nur für die Thematisierung der verschiedenen Standard-Varietäten des Deutschen (vgl. u. a. AMMON: 1995; KNIPF-KOMLÓSI/BEREND: 2001; HÄGI: 2006; FSD: 2007), sondern auch verschiedener Dialekte im Unterricht DaF (innerhalb des deutschen Sprachraumes und des jeweiligen Dialekt-Areals) plädierten (z. B. BASSLER/ SPIEKERMANN: 2001; FEUZ: 2001; STUDER: 2002; ders. 2003).

Mit der starken Orientierung der tschechischen Wirtschaft am Nachbarland Bayern (vgl. Kap. 2.2) erfolgt zugleich die Hinwendung zu einer Region des deutschen Sprachraums, in welcher Dialekte bzw. dialektnahe Varietäten des Deutschen auch im öffentlichen Raum stark verbreitet sind. Angesichts dieses Umstandes liegt es also nahe, die im Alltag benutzten regional (d. h. bairisch, ostfränkisch oder schwäbisch) eingefärbte Regionalsprachen des Freistaats im Rahmen einer Fachsprachenausbildung zu thematisieren, um tschechische DeutschLerner auf eine berufliche Kommunikation mit Partnern aus Bayern vorzubereiten. Die Pionierstudie von BLAHAK (2008) hat diesbezüglich bereits ergeben, dass in den südöstlich an den deutschen Sprachraum angrenzenden Ländern (darunter Tschechien) ein fakultativer Fachsprachenunterricht Deutsch, der angesichts euroregionaler Kooperation auch auf unterer wirtschaftlicher Ebene rezeptive Kompetenzen bezüglich der mundartnahen Varietäten der ,dialektintensiven ‘ Nachbarregionen vermitteln will, eine relevante Lerner-Zielgruppe vorfindet, 
die sich dadurch einen beruflichen Vorteil im wirtschaftlichen Verkehr mit der Nachbarregion verspricht.

\section{Plädoyer für die Institutionalisierung einer studienbegleitenden Zusatzausbildung ,Fachsprache Deutsch mit regionalem Akzent" im tschechischen Hochschulbereich}

Das Gesamtfazit lautet: Tschechien ist ein Land, in dem (zumindest rudimentäre) Deutschkenntnisse nach wie vor weit verbreitet sind. Durch die enge wirtschaftliche Verflechtung mit Deutschland und hier v.a. mit Bayern besteht außerdem ein großer Bedarf an gut ausgebildeten Nachwuchskräften mit Fachsprachen-Kenntnissen in Deutsch, gegebenenfalls mit Bayern-spezifischen regionalsprachlichen wie landeskundlichen Kompetenzen. Diesen Bedarf könnte die akademische Institutionalisierung einer ,Fachsprachenlehre Deutsch mit regionalem Akzent erfolgreich bedienen. Mit der Akkreditierung einer entsprechenden einjährigen studienbegleitenden Zusatzausbildung unter der Bezeichnung BAVARICVM ${ }^{1}$ hat die Pädagogischen Fakultät der Masaryk-Universität Brno in Kooperation mit ihrer bayerischen Partneruniversität in Regensburg als erste staatliche Hochschule Tschechiens diesen Schritt bereits vollzogen.

\section{Literatur}

AMMON (1995): Ammon, Ulrich. Die deutsche Sprache in Deutschland, Österreich und der Schweiz. Das Problem der nationalen Varietäten. Berlin: Walter de Gruyter, 1995.

BLAHAK (2008): Blahak, Boris. Zur Thematisierung regionaler Sprachvarietäten im fachbezogenen Fremdsprachen-Unterricht in Regionen südöstlich des deutschen Sprachraumes, in: Blahak, Boris/Piber, Clemens (Hg.): Deutsch als fachbezogene Fremdsprache in Grenzregionen. Bratislava: Vydavatel'stvo EKONÓM, 2008, 19-37.

CHRIST (2002): Christ, Herbert. Fremdsprachenunterricht in neuen Dimensionen im 21. Jahrhundert, in: Neveling, Christiane (Hg.): Perspektiven für die zukünftige Fremdsprachendidaktik. Tübingen: Gunter Narr, 2002, 25-43.

[DWB] (2008): Presse-Information. Daten zur Wirtschaftsentwicklung in Bayern 2007 im Vergleich zum Bundesgebiet und zu den Ländern. 20. Februar 2008. München: Pressestelle des Bayerischen Staatsministeriums für Wirtschaft, Infrastruktur, Verkehr und Technologie, 2008.

[FSD] (2007): Fremdsprache Deutsch, 2007, 37.

HÄGI (2006): Hägi, Sara. Nationale Varietäten im Unterricht Deutsch als Fremdsprache (= Duisburger Arbeiten zur Sprach- und Kulturwissenschaft 64). Frankfurt a. Main, Berlin, Bern u. a.: Peter Lang, 2006.

JANÍKOVÁ (2008): Janíková, Věra. Einige Anmerkungen zu berufsorientiertem und fachsprachlichem Deutschunterricht aus linguistischer und didaktisch-methodischer Sicht: Medizinische Fachsprache, in: Brünner Heften zu Deutsch als Fremdsprache, 2008, $1,1 / 2,50-62$.

1 Zur detaillierten Fachbeschreibung s. die Homepage des BAVARICVM unter www.bavaricvm. com. 
KNIPF-KOMLÓSI/BEREND (2001): Knipf-Komlósi, Elisabeth/Berend, Nina. Regionale Standards. SprachVariationen in den deutschsprachigen Ländern. Budapest, Pécs: Dialóg Campus Kiadó, 2001.

KOCUMOVÁ (2008): Kocumová, Šárka. Stellung des Deutschen an Grund-, Realschulen und Gymnasien in Brünn. Semesterarbeit. Brno: Masaryk-Universität, 2008.

MATTHEIER (1994): Mattheier, Klaus J. Varietätenzensus. Über die Möglichkeit, die Verbreitung und Verwendung von Sprachvarietäten in Deutschland festzustellen, in: Mattheier, Klaus J./Wiesinger, Peter (Hg.): Dialektologie des Deutschen. Forschungsstand und Entwicklungstendenzen (= Germanistische Linguistik 147). Tübingen: Max Niemeyer, 1994, 413-442.

[MDTHK] (2008): Mitglieder Deutsch-tschechische Industrie- und Handelskammer. Stand: 1. 7. 2008.

NEKULA/ŠICHOVÁ (2004): Nekula, Marek/Šichová, Kateřina. Jakou hodnotu mají jazyky, in: Unternehmenskultur und Unternehmenserfolge, Bd. 1. Prag: Bundesverband Deutscher Unternehmen in der Tschechischen Republik e.V./Goethe-Institut Prag/ Wirtschaftsuniversität Prag, 2004, 253-267.

NEKULA/NEKVAPIL/ŠICHOVÁ (2005): Nekula, Marek/Nekvapil, Jiř̌́/Šichová, Kateřina. Sprachen in multinationalen Unternehmen auf dem Gebiet der Tschechischen Republik (= Forost Arbeitspapiere 31). München: Forschungsverbund Ost- und Südosteuropa, 2005.

STUDER (2003): Studer, Thomas. Varietäten des Deutschen verstehen lernen. Überlegungen und Beobachtungen zum universitären DaF-Unterricht, in: Häcki Suhofer, Annelies (Hg.): Spracherwerb und Lebensalter (= Basler Studien zur deutschen Sprache und Literatur 83). Tübingen, Basel: A. Francke, 2003, 105-118.

VANDERMEEREN (1998): Vandermeeren, Sonja. Fremdsprachen in europäischen Unternehmen. Untersuchungen zu Bestand und Bedarf im Geschäftsalltag mit Empfehlungen für Sprachenpolitik und Sprachunterricht. Waldsteinberg: Heidrun Popp, 1998.

\section{Internetquellen}

[AB] (2005-07): Bayerische Industrie- und Handelskammer/Bayerisches Staatsministerium für Wirtschaft, Infrastruktur, Verkehr und Technologie (Hg.). Der Außenhandel Bayerns. Bavaria's foreign Trade 2004-07, in: Außenwirtschaftsportal Bayern (online), abgerufen am 30. 06. 2009 unter http://www.auwi-bayern.de/awp/inhalte/Arbeitshilfen/ BayernStatistik.html.

BAßLER/SPIEKERMANN (2001): Baßler, Harald/Soekermann, Helmut. Dialekte und Standardsprache im DaF-Unterricht. Wie Schüler urteilen - wie Lehrer urteilen, in: Linguistik online, 2001, 9, 2 (online), abgerufen am 30. 06. 2009 unter: http://linguistikonline.de/9_01/BasslerSpiekermann.html.

[DAF] (2003): Deutsch als Fremdsprache. Erhebung 2000. Berlin, Bonn, Köln, München: StADaF (online), abgerufen am 30. 06. 2009 unter: http://www.goethe.de/mmo/ priv/60112-STANDARD.pdf.

[DAF] (2006): Deutsch als Fremdsprache weltweit. Datenerhebung 2005. Berlin, Bonn, Köln, München: StADaF (online), abgerufen am 30. 06. 2009 unter: http://www.goethe.de/ mmo/priv/1459127-STANDARD.pdf.

[DSD] (2007): Liste der DSD-Schulen in der Tschechischen Republik (online), abgerufen am 30. 06. 2009 unter: www.prag.diplo.de/Vertretung/prag/cs/downloads/liste_dsd__schulen_ d,property=Daten.pdf. 
[EK] (2006): Europäische Kommission (Hg.). Special Eurobarometer 243/Wave 64.3 - TNS Opinion \& Social. Europeans and their Languages (online), abgerufen am 30. 06. 2009 unter: http://ec.europa.eu/public_opinion/archives/ebs/ebs_243_en.pdf.

FEUZ (2001): Feuz, Barbara. Dialektale Varietät als Fremdsprache unterrichten. Ein Erfahrungsbericht, in: Linguistik online, 2001, 9, 2 (online), abgerufen am 30. 06. 2009 unter: http://www.linguistik-online.de/9_01/Feuz.html.

http://bulgarien.ahk.de

http://kroatien.ahk.de

http://tschechien.ahk.de

http://www.bavaricvm.com

http://www.bayhost.de

http://www.bohemicum.de

http://www.bohemicum.de/studium/deutsch-tschechische-studien.html.

http://www.dihk.com.ua

http://www.duihk.hu

http://www.ihk.pl

MARTIN (2006): Martin, Lothar. Tschechien und Bayern: Ausbau der Kooperation und Vorbereitung für den Weltmarkt, in: Radio Praha, 08. 03. 2006 (online), abgerufen am 30. 06. 2009 unter: http://www.tschechien-portal.info/modules.php?op=modload\&nam $\mathrm{e}=$ News \&file $=$ article $\&$ sid $=1175$.

[RVP] (2008): Rámcový vzdělávaci program pro základní vzdělávání (online), abgerufen am 30. 06. 2009 unter: http://www.rvp.cz/soubor/rvpzv.pdf.

SILLNER (2006): Sillner, Bastian. Deutsch als Fremdsprache? - Internationale Sprachenmesse EXPOLINGUA in Prag, in: Radio Praha, 13. 11. 2006 (online), abgerufen am 30. 06. 2009 unter: http://www.radio.cz/de/artikel/852.

STUDER (2002): Studer, Thomas. Dialekte im DaF-Unterricht? Ja, aber ... Konturen eines Konzepts für den Aufbau einer rezeptiven Varietätenkompetenz, in: Linguistik online, 2002, 10, 1 (online), abgerufen am 30. 06. 2009 unter: http://linguistik-online.de/10_ 02/Studer.html.

[WH] (2007): Welthandel 2007 (online), abgerufen am 30. 06. 2009 unter: www.ezv.admin. ch/themen/00504/01530/index.html?download $=M 3 w B P g D B / . . / b K b X r Z 6 l h u D Z z 8 m M p s 2$ gpKfoelang=de.

[WKO] (2007a): Wirtschaftskammer Österreich (Hg.). Warenexporte/Länderstruktur (online), abgerufen am 30. 06. 2009 unter: http://wko.at/statistik/tahzb.htm.

[WKO] (2007b): Wirtschaftskammer Österreich (Hg.). Warenimporte/Länderstruktur (online), abgerufen am 30. 06. 2009 unter: http://wko.at/statistik/tahzb.htm.

[ZFA] (2008): Zentralstelle für das Auslandsschulwesen (Hg.). Auslandsschulverzeichnis (online), abgerufen am 30. 06. 2009 unter: http://www.auslandsschulwesen.de/ cln_115/ nn_388314/Auslandsschulwesen/Auslandsschulverzeichnis/auslandsschulverzeic-node. html?_nnn=true. 